\title{
Preparation and characterization of a novel polysialic acid/gelatin composite hydrogels cross- linked by Tannic acid to improve healing and nursing care of cesarean section dressing
}

\section{Yan Zhang}

Qindao University Medical College Affiliated Yantai Yuhuangding Hospital

\section{Chunjie Wang}

Qindao University Medical College Affiliated Yantai Yuhuangding Hospital

Lijing Wang ( $\sim$ wanglijing541@yahoo.com )

Qindao University Medical College Affiliated Yantai Yuhuangding Hospital https://orcid.org/00000002-9022-7916

\section{Li Hou}

Qindao University Medical College Affiliated Yantai Yuhuangding Hospital

\section{Research Article}

Keywords: Polysialic acid, Gelatin, Tannic acid, Hydrogel, Wound dressing

Posted Date: April 7th, 2021

DOI: https://doi.org/10.21203/rs.3.rs-305430/v1

License: (c) (1) This work is licensed under a Creative Commons Attribution 4.0 International License.

Read Full License 


\section{Abstract}

The infections and delayed wound healing after cesarean delivery is one of the most complicated issues in surgical medicinal field. In the present investigation, designed novel polysialic acid loaded gelatin (PSA-Gel) composite hydrogels cross-linked by tannic acid (TA) has been developed and used as a facile wound dressing to improve cesarean wound healing ability with prevent bactericidal infections. The cross-linking effect was predominant when the TA content was lower, resulting in the formation of a cross-linked network. An effective TA cross-linking effect on the PSA-Gel hydrogel matrix was achieved when the amount of TA was around $15 \mathrm{wt} \%$. The morphology of as-fabricated hydrogels was characterized using scanning electron microscopy (SEM) with an average pore sizes of PSA-Gel, PSA-GelTA-5\%, PSA-Gel-TA-10\%, and PSA-Gel-TA-15\% hydrogels were $95.4 \pm 12.6 \mu \mathrm{m}, 120.4 \pm 8.2 \mu \mathrm{m}, 165.3 \pm 21.6$ $\mu \mathrm{m}$, and $270.2 \pm 32.5 \mu \mathrm{m}$, respectively. The effects of hydrogels on the swelling ratio, in vitro degradation, and mechanical properties were systemically evaluated. The TA cross-linked PSA-Gel hydrogels display strong antimicrobial behavior against gram-positive (Staphylococcus aureus) gram-negative (Escherichia coli) bacteria strains. Moreover, PSA-Gel-TA hydrogels also displayed favorable cytotoxicity toward L929 fibroblast cell lines. Finally, the therapeutic and wound healing potential of the PSA-Gel-TA hydrogels has been studied in vivo using the excision wound model in rats. The results indicate that the PSA-Gel-TA hydrogels have a greater and significant effect on wound closure and increased the wound healing rate compared with native PSA-Gel hydrogels and untreated control group. The findings suggest that PSA-GelTA hydrogels are promising dressing materials for the treatment of wound healing.

\section{Introduction}

The surgical site infections and delayed healing effects after cesarean delivery is a complicated problem past decades. At the same time, many researchers have great attentions on this difficulty and found many interventions intended to reduce infection related issues with administrations of effective antibiotics to progress significant healing effect with addition of skin favored biomaterials. An infection related matters after cesarean surgery is an expensive and it has been projected to cost more than 4,700 USD approximately. Surgical resection is one of the most important means applied in clinical medicine[1-3]. Surgical excision, however, is frequently followed at the insertion wound by postoperative pain, and mostly causes broad normal tissue defects [4]. For example, during tumor excision, which is still a major procedure among several cancer treatment techniques, normal cells across the tumors must be cleared to avoid failure to fully remove tumor cells [5]. Different types of wound care were developed so far, including hydrogels that have already been aimed after to own their 3D porous structure replicating cell membranes, huge water absorption power, and innovative fast encapsulation with other bioactive compounds. In general, injectable hydrogels were considered as prominent prospects for wound dressings, possessing very variable wound sites lacking gel degradation to suit their flexibility, thus promoting surgical activity [6]. A complex biological mechanism is involved during wound healing, containing cascades of activities, like hemostasis, accompanied by inflammation, regeneration, and remodeling [7]. The ideal dressing must be capable of absorbing wound exudate, preserving the wound 
surface area temperature and relative humidity, and getting sufficient antibacterial and anti-inflammatory properties [8].

In biological applications for several years, hydrogels have been studied as drug carrier mechanisms. The 3D porous structure and lipophilicity offer water or blood for hydrogels with characteristics to support and also provide a sustained moist atmosphere for wound healing expatriation [9]. Moreover, due to their soft, wet characteristics and 3D structure identical to the cell membrane, hydrogels are attractive wound dressings. A range of materials such as nanomaterials, natural polymers, semi-synthetic polymers, synthetic polymers, and hydrogels have been used in an attempt to find flexible wound dressings [10-12]. The significant nanomaterial class is polysaccharides from bacteria, such as chitosan, alginate, and polysialic acid (PSA). PSA is an environmentally friendly polysaccharide formed by Escherichia coli fermentation with an alpha-2,8 linkage of $\mathrm{N}$-acetylneuraminic acid (Neu-Ac) [13]. Neu-Ac is commonly distributed in animal and human cells in the oligosaccharide terminal, and PSA can be contained in neural adhesion molecules [14]. Also, PSA-based nanocomposite has been discovered to increase neural cell growth and to deliver beneficial peripheral nerve repair properties as a pathway component through gene expression and in vivo peripheral nerve repair. Collagen, hyaluronic acid, fibrin, sodium alginate, and gelatin are the natural products for bio-ink $[15,16]$. Composite polymers, while generally reduced cell efficiency, have the benefits of abundance, longevity, excellent mechanical properties, and switchable degradability. Gelatin (Gel) is a polymer of low-charge-density peptides that is an ingested collagen substance with a shape close to that of a living cell and has strong biocompatibility [17].

Tannic acid (TA) is a weakly acidic polyphenol containing groups of digal acid conjugated through ester bonds to a core glucose center [18]. In-plant species and micro-organisms, TA components are found and also have the capacity to precipitate molecules (collagen, gelatin, and albumin), certain polysaccharides, and solution alkaloids [19]. TA has significant astringent, oxidative, hemostatic, and antimicrobial activity and, thus, has received desirable applications in the medical fields as a medicine for the treatment of skin ulcers, burns, wounds, and toothache, in addition to other uses in the clothing, wine, and wood factories [20]. The incorporation of TA to other biomaterials is supposed to synergistically improve therapeutic effects. TA treatment has been confirmed to provide many benefits, such as reducing mortality and plasma loss, coagulating with weakened collagen, and providing homeostatic burning effects due to its effective antimicrobial and antioxidant nature [21]. Via hydrogen bonding, TA may be correlated with PSA-Gel chains. In deciding the concomitant mechanical properties, the extent of these transformations and reactions in PSA-Gel-TA systems plays a major role. The creation of a hydrogel matrix that can consistently compete with bacteria, tumor inhibition, and wound healing in medical therapy is therefore of considerable importance [22].

In this study, we combine the advantageous properties of TA, PSA, and Gel to generate novel responsive hydrogels for wound healing and nursing care after cesarean surgery (Scheme 1). The morphology, swelling ratio, in vitro degradation, and mechanical properties of the PSA-Gel-TA hydrogels were characterized. The antibacterial activity of the ternary composite PSA-Gel-TA hydrogels against gramnegative and gram-positive bacteria was investigated. The wound dressing material can efficiently help 
repair and restore the surrounding tissue or cells in the wound. Thus, MTT experiments examined the biocompatibility of PSA-Gel-TA hydrogels and calculated the cell viability percentage of L929 fibroblast cell lines for future wound dressing formulations using the prepared porous PSA-Gel-TA hydrogel. Finally, in vivo studies with rat models were performed with this TA cross-linked PSA-Gel hydrogels.

\section{Experimental Details \\ 2.1. Materials}

Polysialic acid (PSA sodium salt ( $M w=1.6 \times 10^{4} \mathrm{Da}$ ), purchased from Jiangsu Rui Guang Biotechnology Co., Ltd., was further dialyzed against distilled water and lyophilized. Gelatin (Gel), tannic acid (TA) were purchased from Sigma-Aldrich. All reagents have been used without further purification unless otherwise stated. Distilled water was used throughout the experiments.

\subsection{PSA-Gel-TA hydrogel fabrication}

PSA-Gel-TA hydrogels were fabricated briefly, $1.5 \mathrm{~g}$ of PSA was dissolved in $5.0 \mathrm{~mL}$ of $1.0 \% \mathrm{NaOH}$, and $0.5 \mathrm{~mL}$ of epichlorohydrin was added to react at $45^{\circ} \mathrm{C}$ for $12 \mathrm{~h}$ under mixing. Subsequently, a $2.0 \mathrm{gm} \mathrm{Gel}$ in $100 \mathrm{~mL}$ deionized water and heated up to $45^{\circ} \mathrm{C}$ to prepare the polymer solution. After stirring for $2 \mathrm{~h}$, a precipitate formed, which was collected and washed with a large amount of distilled water to give the PSA-Gel hydrogel. Then the different amount of TA solution $(5.0 \mathrm{~mL})$ was added to the PSA-Gel polymer solution prepared previously to achieve the resulting hydrogel solutions with 5,10 , and $15 \mathrm{wt} \%$ TA solution. The final concentration of $15 \% \mathrm{TA}(\mathrm{v} / \mathrm{v})$ was used, and the reaction was heated at $60^{\circ} \mathrm{C}$ for $3 \mathrm{~h}$ to form PSA-Gel-TA solutions. The mixture was further stirred at $60^{\circ} \mathrm{C}$ for $2 \mathrm{~h}$ until a PSA-Gel-TA hydrogel formed.

\subsection{Scanning electron microscope}

The morphology of PSA-Gel and PSA-Gel-TA hydrogels were surface-treated with spray-gold and observed by using a Scanning electron microscope (Quanta 200, FEl, Oregon, USA).

\subsection{Swelling ratio measurements}

The swelling ratio of the PSA-Gel and PSA-Gel-TA hydrogels was carried out according to a general procedure. Weights have been tested for lyophilized hydrogels (W0). The hydrogels were then submerged in PBS ( $\mathrm{pH}=7.4$ ) for up to $3 \mathrm{~h}$. After cleaning the surface water with filter paper, the swelling hydrogels were measured and weighed again after $24 \mathrm{~h}$ of lyophilization. They tested replicated three times of each sample. The swelling ratio of hydrogels was calculated according to the following equations;

Swelling ratio $(\%)=\left(\mathrm{W}_{\mathrm{w}}-\mathrm{W}_{0}\right) / \mathrm{W}_{0} \times 100$.

\subsection{In vitro degradation}

The in vitro degradation of PSA-Gel and PSA-Gel-TA hydrogels was initially processed in a similar way to the swelling ratio analysis. The hydrogels for a given time course were then carefully stored at $20^{\circ} \mathrm{C}$ and 
$37^{\circ} \mathrm{C}$. Weights have been tested for lyophilized hydrogels (W0). The hydrogels PSA-Gel and PSA-Gel-TA were then soaking in PBS ( $\mathrm{pH}=7.4)$ for up to 14 days. The PSA-Gel and PSA-Gel-TA hydrogels were washed properly with DI water to remove any residual contaminants after soaking for $1,4,7,10$, and 14 days and then lyophilized and weighed $(\mathrm{Wd})$. The degradation ratio of hydrogels was determined using the formula;

Degradation ratio $(\%)=\left(\mathrm{W}_{0}-\mathrm{W}_{\mathrm{d}}\right) / \mathrm{W}_{0} \times 100$.

\subsection{Mechanical properties}

Mechanical properties of the hydrogels were tested on the universal tensile strength testing machine SANS CMT7000 with a load rate of $20 \mathrm{~mm} / \mathrm{min}$, equipped with a load cell of $100 \mathrm{~N}$. Specimens of PSAGel and PSA-Gel-TA hydrogel were soaked in water for $2 \mathrm{~h}$ in PBS 7.4. Following the pattern, the samples were taken into a dumbbell shape. The size of the gauge was $500 \mathrm{~mm}$ and the frequency of the axle was $50 \mathrm{~mm} / \mathrm{min}$. From each sample, ten samples were counted. From the stress-strain curve, the individual tensile modulus and fracture strength were obtained.

\subsection{Antibacterial properties}

Antibacterial activity of PSA-Gel and PSA-Gel-TA hydrogels was checked against gram-positive (Staphylococcus aureus) and gram-negative (Escherichia coli) bacteria by using a total colony count method. The test bacteria were inoculated in sterilized TSB and BHI broth, respectively, and incubated for $16 \mathrm{~h}$ at $37^{\circ} \mathrm{C}$. By using fresh broth to obtain a standard inoculum of $106 \mathrm{CFUs} / \mathrm{mL}$ S.aureus and E.coli, the $\mathrm{OD}$ at $595 \mathrm{~nm}$ of each culture was set to 1.0 , which was confirmed by counting the number of colonies on agar media after a 10 -fold dilution sequence. The hydrogel discs were separately put in cultured plates of E.coli and S. aureus and held for $24 \mathrm{~h}$ at $37^{\circ} \mathrm{C}$. The antimicrobial analysis was performed in an aseptic environment after the test samples were put over the sterile cultured solid media, accompanied by incubation at $37^{\circ} \mathrm{C}$ for $1,4,8,24$, and $48 \mathrm{~h}$. At $37^{\circ} \mathrm{C}$ for the bacteria and $30^{\circ} \mathrm{C}$ for the fungus, the plates were incubated for $24 \mathrm{~h}$ and then the number of colonies was measured.

\subsection{Cytotoxicity test}

To test the cytotoxicity of the PSA-Gel and PSA-Gel-TA hydrogels, the L929 mouse fibroblast cell line was used. 90\% DMEM (high glucose), GlutaMAX (TM) and 10\% Fetal Calf Serum (FCS) were prepared for cultivation. In a 96-well microplate, the human fibroblasts were cultured and incubated for $24 \mathrm{~h}$ at $37^{\circ} \mathrm{C}$ and $5 \% \mathrm{CO}_{2}$ in a humidified atmosphere. Cultured monolayer washed with PBS at $90 \%$ confluence, then trypsinized with $2.0 \mathrm{ml}(0.5 \%)$ of the trypsin-EDTA solution, incubated for $2 \mathrm{~min}$. The viability of the cells was assessed using an MTT assay. In brief, at a concentration of $0.5 \mathrm{mg} / \mathrm{mL}$, MTT with culture medium was dissolved. Samples were extracted and MTT solution was applied (100 $\mu \mathrm{L} /$ well) into each well and incubated for $2 \mathrm{~h}$ at $37^{\circ} \mathrm{C}$. The medium was replaced with DMSO $(100 \mu \mathrm{L})$ after incubation. On the distilled hydrogel, the cells with a concentration of $3 \times 10^{4}$ were inoculated and cultured for $24 \mathrm{~h}$. The cells in the culture medium were the negative influence of the experiment on days $1,7,14$ and 21 . The 
percentage of cytotoxicity was calculated by Eq. (3) as abs (sample) is the absorbance of the treated sample and abs (control) is the absorbance of control.

Cell viability $(\%)=\left(\right.$ abs $_{(\text {sample })} / \mathrm{abs}_{(\text {control })} \times 100 \%$ (3)

\subsection{Animal experiment}

The animals were purchased from the Yantai Yuhuangding Hospital of Qingdao University, and male Sprague Dawley rats of $4 \sim 6$ weeks old were used in the study. Animals were maintained and fed ad libitum under strict pathogen-free conditions. Animals had access to regular chow and water ad libitum at all times and all procedures were carried out with the prior approval of the Institutional Animal Care and Use Committee of Qingdao University Yantai Yuhuangding Hospital. The dressings were cross-linked, dried gel in the in vivo model that could be cut for use. The rats were randomly assigned to 4 groups according to the samples with 3 rats in each group: control (1×PBS solution), PSA-Gel, and PSA-Gel-TA hydrogels. Anesthetized rats were sterilized with iodine wine under sterile conditions and a $1.5 \mathrm{~cm}$ (width) area of about $5 \mathrm{~cm}$ (length) was marked on the dorsal area of the rats, which was the spontaneous flap area determined (note: the pedicel of the flap was in the tail end). A full-thick skin flap was raised, the soft tissue underneath was cut off from the flap, and the pedicle would cut off any axial blood vessels going through the flap. The flap was carefully sutured back to its original position, $0.25 \mathrm{~mL}$ samples and 0.25 $\mathrm{mL}$ bacterial suspension (S. aureus, OD600 $=0.1$ ) were injected intradermally into each flap from the distal end to the pedicle end (syringe specifications $/ \mathrm{mm}$ : $0.45 \times 16$ ). After that, the rats were put back to their cages after recovering from anesthesia. The dressings were impassive after surgery on days 7,14 and 21, and the wounds were inspected and image for wound size measurements. The percentage of wound closure was determined using the following equation at each point in time:

Wound contraction (\%) = Day 0 - Day $x /$ Day 0 x $100 \%$

which $\mathrm{x}$ is the corresponding timepoint. The skin wound tissue was excised after imaging, fixed with $10 \%$ formalin, and stained with hematoxylin and eosin to perform a histological assessment.

\subsection{Statistical analysis}

Statistical analysis was performed for post hoc correlations in Origin 8.5 by one-way ANOVA followed by Tukey's HSD (Microcal Software Inc., Northampton, MA, USA). A t-test was conducted to compare the importance of the disparity between the means of the two groups. A p value $<0.05$ was measured statistically significant.

\section{Results And Discussions}

\subsection{Morphology of PSA-Gel and PSA-Gel-TA hydrogels}

Scaffold microstructure and porosity are very important in bone tissue engineering applications. The space for nutrient distribution, cell formation, and propagation for signaling pathways and stimulation 
can be given by a porous structure [23]. Besides, for cell compatibility and development, surface morphology is important. SEM was used to examine the microstructure and morphology of freeze-dried PSA-Gel and PSA-Gel-TA hydrogels. The SEM images demonstrate that the hydrogels were porous, obviously seen with closed pores with accessible and interconnected pores [24]. The morphologies of PSA-Gel hydrogels of different concentrations of TA $(5,10$, and $15 \%)$ are shown in Fig. 1. The pores contained in PSA-Gel-TA were much larger than commonly seen in hydrogels containing PSA-Gel, so the high porous structures were predominantly supported by TA molecules [25]. The wall thickness and pore size of PSA-Gel-TA hydrogels have also improved with the growth of TA. The amount of TA content is strongly determined by the degree of crosslinking, where the denser structure with lower porosity is the more crosslinking in the hydrogels. Thus, in enhancing the porous structure and surface morphology of PSA-Gel hydrogels, TA plays a major role.

The average pore sizes obtained from the SEM images were $95.4 \pm 12.6 \mu \mathrm{m}, 120.4 \pm 8.2 \mu \mathrm{m}, 165.3 \pm 21.6$ $\mu \mathrm{m}$, and $270.2 \pm 32.5 \mu \mathrm{m}$ for PSA-Gel, PSA-Gel-TA-5\%, PSA-Gel-TA-10\%, and PSA-Gel-TA-15\% hydrogels surfaces, respectively (Fig. 1). Average pore sizes vary from 50 to $710 \mu \mathrm{m}$ for bone tissue regeneration. Many studies have indicated that macroporous structures ranging from 150 to $300 \mu \mathrm{m}$ are favorable for the healing of bones. The pore size range of the PSA-Gel-TA hydrogels was $150-300 \mu \mathrm{m}$, which is sufficient for the bone tissue. 15\% TA in PSA-Gel was more desirable, with pore sizes of $120-270 \mu \mathrm{m}$ supporting bacterial activity and the development of fibroblast cells, based on the pore sizes of the lyophilized hydrogels [26].

\subsection{Mechanical properties}

The mechanical property of PSA-Gel and PSA-Gel-TA hydrogels is of great importance for the application in tissue engineering as shown in Fig. 2. Due to the regular motion and bending of joints, conventional hydrogel dressings are prone to elongation, wear, and even injury. The self-healing hydrogels could therefore significantly extend their service time as a current wound dressing [27, 28]. Figure 2a,b shows that the strain-stress curves and compressive strength of PSA-Gel and PSA-Gel-TA hydrogel present an upward trend. The tensile strength and compressive strength of PSA-Gel are $56.3 \mathrm{kPa}$ and $69.5 \%$, respectively [28]. After the introduction of TA, the tensile strength and compressive strength of PSA-GelTA-15\% increases by $76.4 \mathrm{kPa}$ and $79.8 \%$ comparing to PSA-Gel, which might be due to the reason that the TA increases the flexibility of the coating. It can be seen that the mechanical properties of PSA-Gel-TA hydrogels are much improved by further ionic crosslinking [29]. Therefore, the hydrogels are strong enough for cell culture in tissue engineering. Moreover, the young's modulus of the PSA-Gel-TA hydrogel increased from $\sim 13.4 \mathrm{kPa}$ to $\sim 42.5, \sim 55.1$, and $\sim 73.6 \mathrm{kPa}$ when the concentrations of incorporated TA were $0,5,10$, and $15 \%$, respectively. An approximately 6 -fold increase in young's modulus could be observed in the PSA-Gel-TA-15\% hydrogel in comparison to the PSA-Gel hydrogel. If there is less hydrogel in the young modulus, cellular anchorage can be repelled and the encapsulated cells can expand into spheres [30].

\subsection{Swelling ratio and degradation}


The swelling ratio (SR) is one of the most important parameters for evaluating PSA-Gel and PSA-Gel-TA hydrogels. Swelling experiments under different conditions were performed to determine the effect of TA on the swelling action of PSA-Gel-TA hydrogels. Firstly, SR relationship with time was examined in Fig. 3a demonstrates PSA-Gel and PSA-Gel-TA hydrogel swelling kinetics in PBS $(\mathrm{pH}=7.4)$. It can be shown that these hydrogels have an increasing swelling activity that swells rapidly in the first $5 \mathrm{~h}$, then rises slowly after $10 \mathrm{~h}$, and after around $36 \mathrm{~h}$ of swelling, the hydrogel eventually reaches equilibrium [31]. Hydrogels have an equilibrium swelling ratio (SR) of $13.6-32.5 \%$. This result can be due to the greater quantity of TA strongly interacting with the PSA-Gel hydrogel functional groups, which decreased the swelling potential. The findings suggest that crosslinking effectively prevents PSA-Gel-TA hydrogel swelling to retain the original structure. The degradation of hydrogels is significant in bone tissue engineering applications, in addition to a swelling of the hydrogels. Figure $3 \mathrm{~b}$ demonstrates the PSA-Gel and PSA-GelTA hydrogels degradation activity. The findings suggest that the hydrogels degrade slowly and spontaneously. Hydrogels also maintain more than $89.4 \%$ weight after 21 days and preserve the original form. The findings suggest that if used in tissue engineering, the hydrogels would allow the tissue enough time to restore [32]. PSA-Gel-TA hydrogels have the potential for bone tissue engineering applications from the view of pore size distribution, swelling, degradation, and mechanical properties of hydrogels.

\subsection{Antibacterial analysis}

The effects of PSA-Gel and PSA-Gel-TA hydrogels against two common pathogenic microorganisms, E. coli, and S. aureus, are depicted in Fig. 4. The total number of bacteria treated by hydrogels has decreased compared with regulation. PSA-Gel hydrogels were more effective than E.coli in inhibiting the growth of S. aureus, while PSA-Gel hydrogels loaded with TA showed increased activity against both bacteria. As shown in Fig. 4, PSA-Gel-TA hydrogel demonstrated excellent antibacterial effects, killing over $92 \%$ of bacteria [33]. Compared to the control group, there were more bacterial colonies in the PSA-Gel and PSA-Gel-TA hydrogels classes, suggesting that the hydrogel setting is more acceptable than bacterial growth control, without the intervention of TA we used could not kill more bacteria [34]. TA may cause Gram-positive bacteria to be bacteriostatic because it hydrolyses the cell wall. It was thought that TA stacks at the bacterial cell surface and interferes with the metabolism of bacteria or penetrates the cell membrane and then adsorbs into biomolecules of nucleic acid [35]. Other hydrogels without crosslinking, however, have failed to inhibit bacterial growth, which indicates that TA has antibacterial activity in the PSA-Gel-TA hydrogel. These studies indicate the important antimicrobial effects of PSA-Gel-TA hydrogels against both bacteria (E.coli and S. aureus).

\subsection{Cytotoxicity of the hydrogels}

The in vitro biocompatibility of the hydrogels was assessed using MTT assay with L929 cells (Fig. 5). With the rise in TA levels results in a higher level of crosslinking, the viability of the cells was significantly precious and decreased properly. Remarkably, PSA-Gel-TA-5\%, PSA-Gel-TA-10\%, and PSA-Gel-TA-15\% of all preserved attractive great cell viabilities, which were even higher than PSA-Gel, Gel, TA of about 115\% 
and $142 \%$. The TA solution alone was analyzed and the L929 cell viability was also higher than the blank control. To facilitate human fibroblast proliferation, TA has been confirmed, so the presence of TA in PSAGel hydrogels could indeed accelerate fibroblast formation [36]. Finally, by considering previously recorded physical parameters, TA $15 \%$ was chosen for in vivo experiments, but most significantly, with good cell viability results presenting no deviation from the blank control. The highest cytotoxicity of the PSA-Gel-TA-15\% hydrogels was assessed after 1, 7, 14, and 21 days by performing the MTT assay. L929 cells grown on day 21 in PSA-Gel-TA-15\% hydrogels had enhanced viability compared with those grown on days 1 to 14 . On day 21, the GEL-SS-PVA matrices showed significantly greater cellular activity than on days 1, 7 and 14. Compared with controls, the cell viability of each treatment group was statistically significant $(p<0.05)$. In the proposed approach, the development of GEL-SS-PVA hydrogels from cell viability can be used as a loaded TA, regarded as a potential candidate for bone regeneration in biological applications [37].

\subsection{In vivo wound healing study}

The smart design of the PSA-Gel and PSA-Gel-TA hydrogels enables it to be superior in promoting wound healings through eliminating bacterial infection and promoting hair follicles/vessel regeneration. As shown in Fig. 6. By injecting this hydrogel into the infected skin flap wound of a rat model, we tested the wound healing effect of PSA-Gel and PSA-Gel-TA hydrogels. By examining the wound area, clear variations and reductions in wound reduction were observed and measured. Wounds treated with PSA-Gel and PSA-Gel-TA hydrogels accelerated wound reduction as related with the control from day 7 after wound healing, in precise the results of PSA-Gel-TA hydrogels were considerably higher than the control and PSA-Gel hydrogels [38]. As shown, the wound with PSA-Gel-TA hydrogels exhibited excellent healing effects without obvious infection compared to the wound treated with blank PSA-Gel, which indicates the powerful antibacterial activity of the TA, rendering anti-infection feasible $[39,40]$. Of note, the in vivo swelling of the hydrogels is lower than in vitro studies. The degree of wound contraction of PSA-Gel-TA hydrogels was significantly higher than PSA-Gel hydrogels with little statistical variance. The control final wound reductions, PSA-Gel and PSA-Gel-TA hydrogels were $65 \%, 73 \%$ and $94 \%$ respectively at day 21 .

Although the fact that none of the wounds were fully healed by day 21 , as seen in the photograph, closure of the wound treated with PSA-Gel-TA hydrogels had almost existed. On the other hand, with the treatment of PSA-Gel-TA hydrogels, no apparent inflammation in the wound is found over 7 days and the wound improves well within 14 days.

To further check the healing status of the skin wounds, we selected the wounds with the PBS control, blank PSA-Gel hydrogel, PSA-Gel-TA hydrogel at days 7, 14, and 21 to observe their tissue section, which was realized through H\&E staining are shown in Fig. 7. After 14 days of wound treatment, the newly established extracellular matrix over scar tissue reported at a greater rate the aggregation of inflammatory cells, fibroblasts, and collagen bundles [41]. The inflammatory response was observed on day 7 to varying degrees in all groups; the development of granulation materials, with all the creation of new small blood vessels and hair follicles, in the wound treated with PSA-Gel-TA hydrogels [38, 42]. Epidermal layer regeneration was understood in all groups from day 14 onwards. The layers of the newly 
regenerated epidermal cells were relatively smooth in the existence of PSA-Gel-TA hydrogels as wound dressings, but the untreated groups newly reinforced epidermis appeared to be stained. Also, more blood vessel restoration and hair follicle development were found in the wounds of treated animals with PSAGel-TA hydrogels compared to the untreated community. Both groups displayed a strong epidermis with thick epithelia, hair follicles, and fibroblast-forming dense distribution of collagen fibers and freshly formed, efficient blood vessels that exhibited almost complete wound healing. In contrast, due to various their gelling properties, hydrogels are ideal materials for covering irregular wound surfaces. The key mechanism in the tissue matrix is wound contraction, which can decrease the healing time by regenerating skin cells to restore damaged tissue at the wound site [43]. The most critical stage is the synthesis and deposition of collagen, remodeling, and maturation during tissue repair and regeneration. Inflammation in wounds, often in the absence of an infection that affects the effects of wound healing, is a fundamental aspect. TA is an important component of natural antioxidants that can be used as food preservatives or nutraceuticals but has been shown to potentiate the action of oxidant-induced PSA-Gel hydrogels and promote wound regeneration $[36,44,45]$. The results indicate that the design of PSA-GelTA hydrogels has been able to preserve TA antioxidant activity without impairing the proper inflammation of the wound. The newly developed epidermal tissues could develop on the hydrogels in the presence of PSA-Gel-TA hydrogels as wound dressings, results in smooth and less wrinkled patterns as seen in the histological assessment, also allowing for the prompt restoration of vascular and tissue. However, using this current rat model, the excellent ability of PSA-Gel-TA hydrogels, TA15\% in particular, to improve wound recovery is demonstrated in vivo. Furthermore, these findings suggest that for wound healing, the hydrogel can be a healthy therapeutic material.

\section{Conclusions}

A novel TA cross-linked PSA-Gel hydrogels were investigated in the view of a potential dressing material with enhanced wound regeneration. We have successfully prepared a PSA-Gel hydrogels system amino groups into a 3D network by incorporating TA with the surface of PSA-Gel hydrogels. The highly porous structure of PSA-Gel-TA hydrogels with an average pore size of $270.2 \pm 32.5 \mu \mathrm{m}$ was revealed in the SEM picture. In several newspapers, the formulated hydrogel membranes demonstrated good stability, had a good swelling ratio, in vitro degradation, and mechanical properties. More significantly, by killing the rigidly adhere bacteria on the hydrogel surface with a high concentration of TA, the PSA-Gel-TA hydrogels demonstrated superior antibacterial activity against both gram-positive and gram-negative bacteria and had excellent biocompatibility by improving cell proliferation. The key reason for its promotion of wound healing is its antimicrobial, cell proliferation, and anti-inflammatory effects, as well as the control of various wound healing factors. The prepared PSA-Gel-TA hydrogel membranes showed the desired results in healing when applied in a rat model to test the healing effects on the wound. These findings indicate that hydrogels from TA and PSA-Gel play a synergistic function in wound healing, and hydrogels from TA-loaded PSA-Gel are promising candidates for skin regeneration wound dressing. There are still difficulties in applying them for wound healing applications, considering the potential impact of multifunctional hydrogels. For future research, it may be a promising approach to build advanced 
multifunctional hydrogels by incorporating wound monitoring tools such as pathogenic infection detectors and smart drug release systems at the same time.

\section{References}

[1] A.M. Buresch, A. Van Arsdale, M. Ferzli, N. Sahasrabudhe, M. Sun, J. Bernstein, P.S. Bernstein, I.M. Ngai, D.J. Garry, Comparison of Subcuticular Suture Type for Skin Closure after Cesarean Delivery: A Randomized Controlled Trial, Obstet. Gynecol. 130 (2017) 521-526. https://doi.org/10.1097/AOG.0000000000002200.

[2] A.M. Valent, C. Dearmond, J.M. Houston, S. Reddy, H.R. Masters, A. Gold, M. Boldt, E. Defranco, A.T. Evans, C.R. Warshak, Effect of post-cesarean delivery oral cephalexin and metronidazole on surgical site infection among obese women: A randomized clinical trial, JAMA - J. Am. Med. Assoc. 318 (2017) 10261034. https://doi.org/10.1001/jama.2017.10567.

[3] M.G. Tuuli, J. Liu, A.T.N. Tita, S. Longo, A. Trudell, E.B. Carter, A. Shanks, C. Woolfolk, A.B. Caughey, D.K. Warren, A.O. Odibo, G. Colditz, G.A. MacOnes, L. Harper, Effect of prophylactic negative pressure wound therapy vs standard wound dressing on surgical-site infection in obese women after cesarean delivery: A randomized clinical trial, JAMA - J. Am. Med. Assoc. 324 (2020) 1180-1189. https://doi.org/10.1001/jama.2020.13361.

[4] I. Power, Recent advances in postoperative pain therapy, Br. J. Anaesth. 95 (2005) 43-51. https://doi.org/10.1093/bja/aei037.

[5] L. Yang, P. Shi, G. Zhao, J. Xu, W. Peng, J. Zhang, G. Zhang, X. Wang, Z. Dong, F. Chen, H. Cui, Targeting cancer stem cell pathways for cancer therapy, Springer US, 2020.

https://doi.org/10.1038/s41392-020-0110-5.

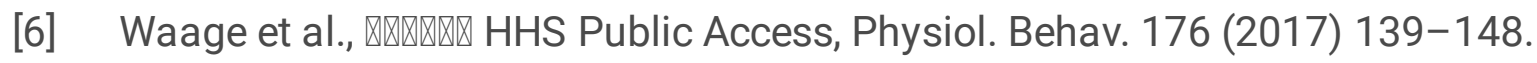

https://doi.org/10.1016/j.addr.2018.03.007.In.

[7] A.C.D.O. Gonzalez, Z.D.A. Andrade, T.F. Costa, A.R.A.P. Medrado, Wound healing - A literature review, An. Bras. Dermatol. 91 (2016) 614-620. https://doi.org/10.1590/abd1806-4841.20164741.

[8] J.M. Reinke, H. Sorg, Wound repair and regeneration, Eur. Surg. Res. 49 (2012) 35-43. https://doi.org/10.1159/000339613.

[9] G. Lokhande, J.K. Carrow, T. Thakur, J.R. Xavier, M. Parani, K.J. Bayless, A.K. Gaharwar, Nanoengineered injectable hydrogels for wound healing application, Acta Biomater. 70 (2018) 35-47. https://doi.org/10.1016/j.actbio.2018.01.045.

[10] M. Mir, M.N. Ali, A. Barakullah, A. Gulzar, M. Arshad, S. Fatima, M. Asad, Synthetic polymeric biomaterials for wound healing: a review, Prog. Biomater. 7 (2018) 1-21. 
https://doi.org/10.1007/s40204-018-0083-4.

[11] Y. Zhong, H. Xiao, F. Seidi, Y. Jin, Natural Polymer-Based Antimicrobial Hydrogels without Synthetic Antibiotics as Wound Dressings, Biomacromolecules. 21 (2020) 2983-3006. https://doi.org/10.1021/acs.biomac.0c00760.

[12] G.D. Mogoşanu, A.M. Grumezescu, Natural and synthetic polymers for wounds and burns dressing, Int. J. Pharm. 463 (2014) 127-136. https://doi.org/10.1016/j.ijpharm.2013.12.015.

[13] B.X. Lin, Y. Qiao, B. Shi, Y. Tao, Polysialic acid biosynthesis and production in Escherichia coli: current state and perspectives, Appl. Microbiol. Biotechnol. 100 (2016) 1-8. https://doi.org/10.1007/s00253-015-7019-x.

[14] R.L. Schnaar, R. Gerardy-Schahn, H. Hildebrandt, Sialic acids in the brain: Gangliosides and polysialic acid in nervous system development, stability, disease, and regeneration, Physiol. Rev. 94 (2014) 461-518. https://doi.org/10.1152/physrev.00033.2013.

[15] J.S. Kim, S. Hong, C. Hwang, Bio-ink Materials for 3D Bio-printing, J. Int. Soc. Simul. Surg. 3 (2016) 49-59. https://doi.org/10.18204/jissis.2016.3.2.049.

[16] J. Gopinathan, I. Noh, review 52018 Recent trends in bioinks for 3D printing.pdf, Biomater. Res. (2018) 1-15.

[17] D. Liu, M. Nikoo, G. Boran, P. Zhou, J.M. Regenstein, Collagen and gelatin, Annu. Rev. Food Sci. Technol. 6 (2015) 527-557. https://doi.org/10.1146/annurev-food-031414-111800.

[18] F. Weber, E. Sagstuen, Q.Z. Zhong, T. Zheng, H. Tiainen, Tannic Acid Radicals in the Presence of Alkali Metal Salts and Their Impact on the Formation of Silicate-Phenolic Networks, ACS Appl. Mater. Interfaces. 12 (2020) 52457-52466. https://doi.org/10.1021/acsami.0c16946.

[19] X.P. Liao, Z.B. Lu, B. Shi, Selective adsorption of vegetable tannins onto collagen fibers, Ind. Eng. Chem. Res. 42 (2003) 3397-3402. https://doi.org/10.1021/ie0209475.

[20] A. Pizzi, Tannins: Prospectives and actual industrial applications, Biomolecules. 9 (2019). https://doi.org/10.3390/biom9080344.

[21] B. Kaczmarek, Tannic acid with antiviral and antibacterial activity as a promising component of biomaterials-A minireview, Materials (Basel). 13 (2020). https://doi.org/10.3390/ma13143224.

[22] and R.V.J. Lori M. Millner, Mark W. Linder, 139-148. https://doi.org/10.1016/j.addr.2018.04.008.Drug.

[23] B.J. Lawrence, S. V. Madihall, Cell colonization in degradable 3D porous matrices Basics of Porous Structures Importance of Spatial Architecture, Cell Adh. Migr. 2 (2008) 9-16. 
[24] M.S. Rahman, M.M. Islam, M.S. Islam, A. Zaman, T. Ahmed, S. Biswas, S. Sharmeen, T.U. Rashid, M.M. Rahman, Morphological Characterization of Hydrogels, 2019. https://doi.org/10.1007/978-3-31977830-3_28.

[25] Y.N. Chen, C. Jiao, Y. Zhao, J. Zhang, H. Wang, Self-Assembled Polyvinyl Alcohol-Tannic Acid Hydrogels with Diverse Microstructures and Good Mechanical Properties, ACS Omega. 3 (2018) 1178811795. https://doi.org/10.1021/acsomega.8b02041.

[26] S.J. Shirbin, F. Karimi, N.J.A. Chan, D.E. Heath, G.G. Qiao, Macroporous Hydrogels Composed Entirely of Synthetic Polypeptides: Biocompatible and Enzyme Biodegradable 3D Cellular Scaffolds, Biomacromolecules. 17 (2016) 2981-2991. https://doi.org/10.1021/acs.biomac.6b00817.

[27] I. Hussain, G. Fu, Self-healing hydrogels, 2020. https://doi.org/10.1016/b978-0-12-818450-9.000131.

[28] J. Wang, F. Tang, Y. Wang, Q. Lu, S. Liu, L. Li, Self-Healing and Highly Stretchable Gelatin Hydrogel for Self-Powered Strain Sensor, ACS Appl. Mater. Interfaces. 12 (2020) 1558-1566. https://doi.org/10.1021/acsami.9b18646.

[29] J. Wen, X. Zhang, M. Pan, J. Yuan, Z. Jia, L. Zhu, A robust, tough and multifunctional polyurethane/tannic acid hydrogel fabricated by physical-chemical dual crosslinking, Polymers (Basel). 12 (2020). https://doi.org/10.3390/POLYM12010239.

[30] H.F. Pastrana, A.X. Cartagena-Rivera, A. Raman, A. Ávila, Evaluation of the elastic Young's modulus and cytotoxicity variations in fibroblasts exposed to carbon-based nanomaterials, J. Nanobiotechnology. 17 (2019) 1-15. https://doi.org/10.1186/s12951-019-0460-8.

[31] R.A. McBath, D.A. Shipp, Swelling and degradation of hydrogels synthesized with degradable poly( $\beta$-amino ester) crosslinkers, Polym. Chem. 1 (2010) 860-865. https://doi.org/10.1039/c0py00074d.

[32] J. Zhu, R.E. Marchant, Design properties of hydrogel tissue-engineering scaffolds, Expert Rev. Med. Devices. 8 (2011) 607-626. https://doi.org/10.1586/erd.11.27.

[33] S. Li, S. Dong, W. Xu, S. Tu, L. Yan, C. Zhao, J. Ding, X. Chen, Antibacterial Hydrogels, Adv. Sci. 5 (2018). https://doi.org/10.1002/advs.201700527.

[34] G. Dong, H. Liu, X. Yu, X. Zhang, H. Lu, T. Zhou, J. Cao, Antimicrobial and anti-biofilm activity of tannic acid against Staphylococcus aureus, Nat. Prod. Res. 32 (2018) 2225-2228. https://doi.org/10.1080/14786419.2017.1366485.

[35] H. Bai, Y. You, H. Yan, J. Meng, X. Xue, Z. Hou, Y. Zhou, X. Ma, G. Sang, X. Luo, Antisense inhibition of gene expression and growth in gram-negative bacteria by cell-penetrating peptide conjugates of peptide nucleic acids targeted to rpoD gene, Biomaterials. 33 (2012) 659-667. https://doi.org/10.1016/j.biomaterials.2011.09.075. 
[36] Y. Chen, L. Tian, F. Yang, W. Tong, R. Jia, Y. Zou, L. Yin, L. Li, C. He, X. Liang, G. Ye, C. Lv, X. Song, Z. Yin, Tannic Acid Accelerates Cutaneous Wound Healing in Rats Via Activation of the ERK 1/2 Signaling Pathways, Adv. Wound Care. 8 (2019) 341-354. https://doi.org/10.1089/wound.2018.0853.

[37] B. Liu, Y. Wang, Y. Miao, X. Zhang, Z. Fan, G. Singh, X. Zhang, K. Xu, B. Li, Z. Hu, M. Xing, Biomaterials Hydrogen bonds autonomously powered gelatin methacrylate hydrogels with superelasticity , self-heal and underwater self- adhesion for sutureless skin and stomach surgery and E-skin, Biomaterials. 171 (2018) 83-96. https://doi.org/10.1016/j.biomaterials.2018.04.023.

[38] X. He, X. Liu, J. Yang, H. Du, N. Chai, Z. Sha, M. Geng, X. Zhou, C. He, Tannic acid-reinforced methacrylated chitosan/methacrylated silk fibroin hydrogels with multifunctionality for accelerating wound healing, Carbohydr. Polym. 247 (2020). https://doi.org/10.1016/j.carbpol.2020.116689.

[39] S.R. Tintino, C.D.M. Oliveira-Tintino, F.F. Campina, R.L.P. Silva, M. do S. Costa, I.R.A. Menezes, J.T. Calixto-Júnior, J.P. Siqueira-Junior, H.D.M. Coutinho, T.C. Leal-Balbino, V.Q. Balbino, Evaluation of the tannic acid inhibitory effect against the NorA efflux pump of Staphylococcus aureus, Microb. Pathog. 97 (2016) 9-13. https://doi.org/10.1016/j.micpath.2016.04.003.

[40] R.S. Carvalho, C.A. Carollo, J.C. de Magalhães, J.M.C. Palumbo, A.G. Boaretto, I.C. Nunes e Sá, A.C. Ferraz, W.G. Lima, J.M. de Siqueira, J.M.S. Ferreira, Antibacterial and antifungal activities of phenolic compound-enriched ethyl acetate fraction from Cochlospermum regium (mart. Et. Schr.) Pilger roots: Mechanisms of action and synergism with tannin and gallic acid, South African J. Bot. 114 (2018) 181187. https://doi.org/10.1016/j.sajb.2017.11.010.

[41] L.E. Tracy, R.A. Minasian, E.J. Caterson, Extracellular Matrix and Dermal Fibroblast Function in the Healing Wound, Adv. Wound Care. 5 (2016) 119-136. https://doi.org/10.1089/wound.2014.0561.

[42] P. Taheri, R. Jahanmardi, M. Koosha, S. Abdi, Physical, mechanical and wound healing properties of chitosan/gelatin blend films containing tannic acid and/or bacterial nanocellulose, Int. J. Biol. Macromol. 154 (2020) 421-432. https://doi.org/10.1016/j.ijbiomac.2020.03.114.

[43] T. Klaus, S. Takano, G. Winnewisser, Laboratory measurement of the $\mathrm{N}=1 \leftarrow 0$ rotational transition of $\mathrm{NH}$ at $1 \mathrm{THz}$, Astron. Astrophys. 322 (1997) 1-36.

https://doi.org/10.1126/scitranslmed.3009337.Wound.

[44] P. Orlowski, M. Zmigrodzka, E. Tomaszewska, K. Ranoszek-Soliwoda, M. Czupryn, M. Antos-Bielska, J. Szemraj, G. Celichowski, J. Grobelny, M. Krzyzowska, Tannic acid-modified silver nanoparticles for wound healing: The importance of size, Int. J. Nanomedicine. 13 (2018) 991-1007. https://doi.org/10.2147/IJN.S154797.

[45] N. Ninan, A. Forget, V.P. Shastri, N.H. Voelcker, A. Blencowe, Antibacterial and Anti-Inflammatory pHResponsive Tannic Acid-Carboxylated Agarose Composite Hydrogels for Wound Healing, ACS Appl. Mater. Interfaces. 8 (2016) 28511-28521. https://doi.org/10.1021/acsami.6b10491. 


\section{Figures}
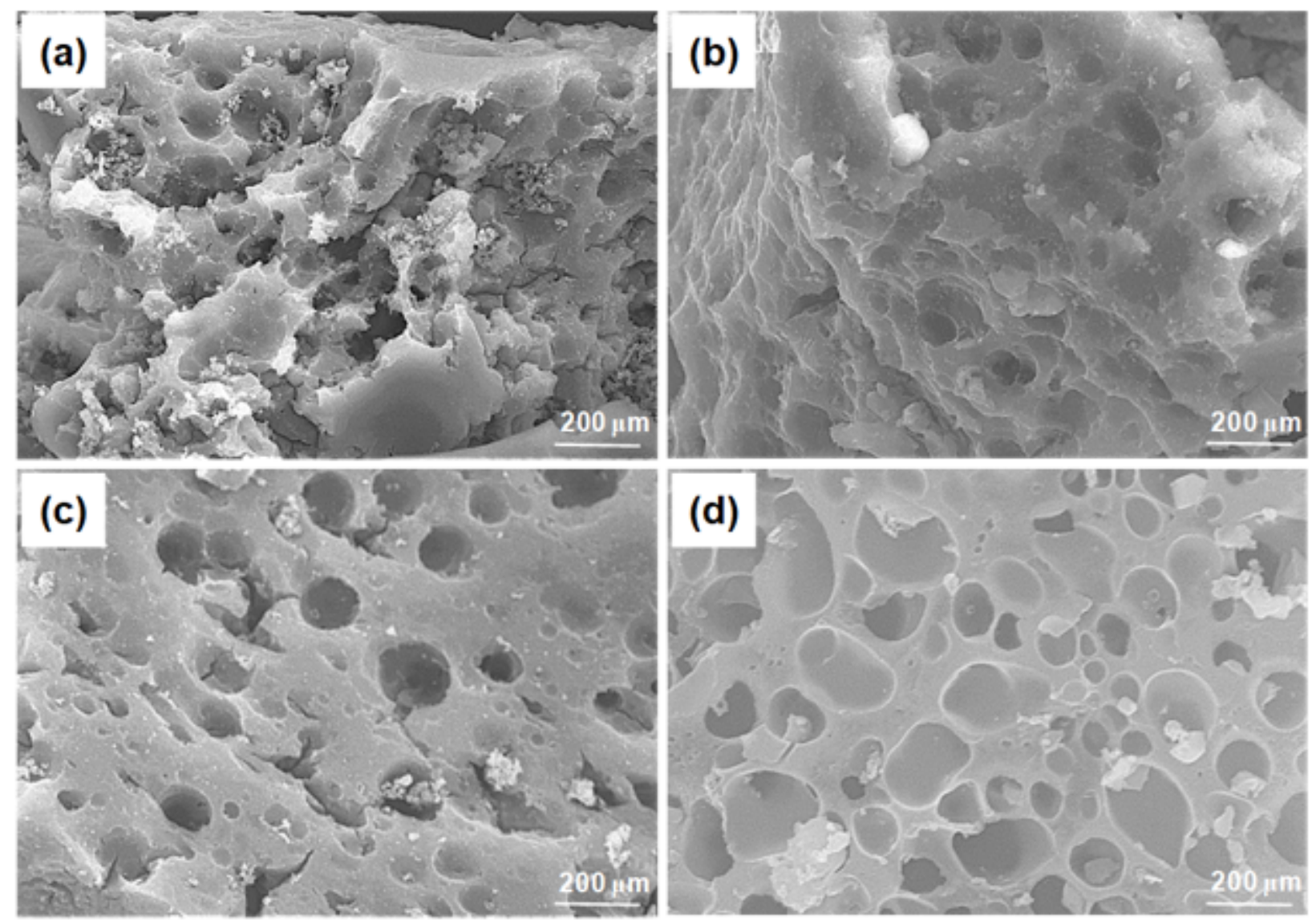

\section{Figure 1}

SEM images of PSA-Gel (a), PSA-Gel-TA-5\% (b), PSA-Gel-TA-10\% and (c), and PSA-Gel-TA-15\% hydrogel (d).
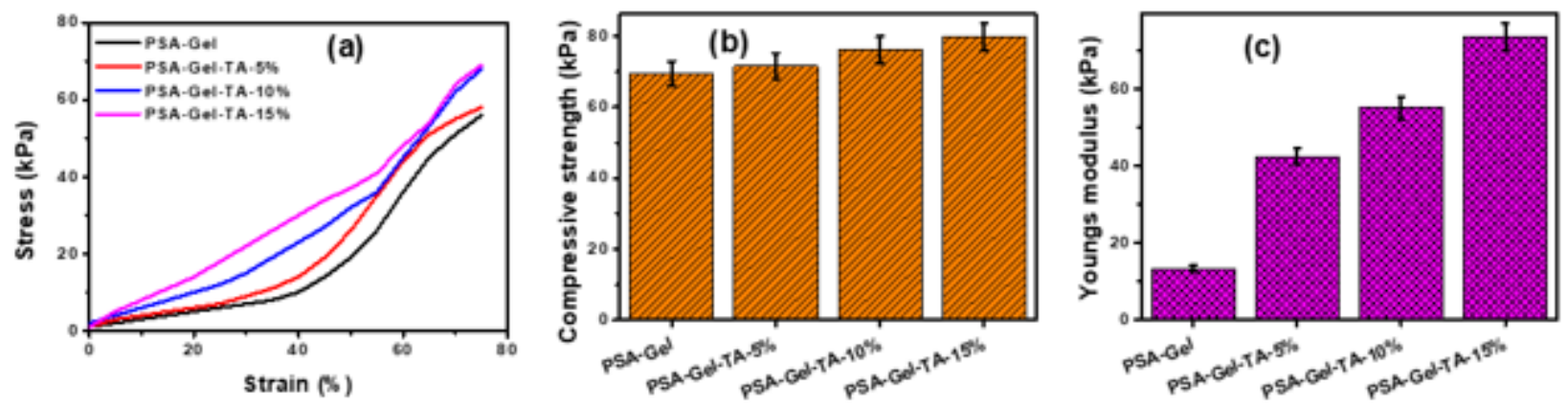

Figure 2

(a) Strain-stress curves, (b) compressive strength, and (c) Youngs modulus of the PSA-Gel, PSA-Gel-TA$5 \%$, PSA-Gel-TA-10\%, and PSA-Gel-TA-15\% hydrogels. 

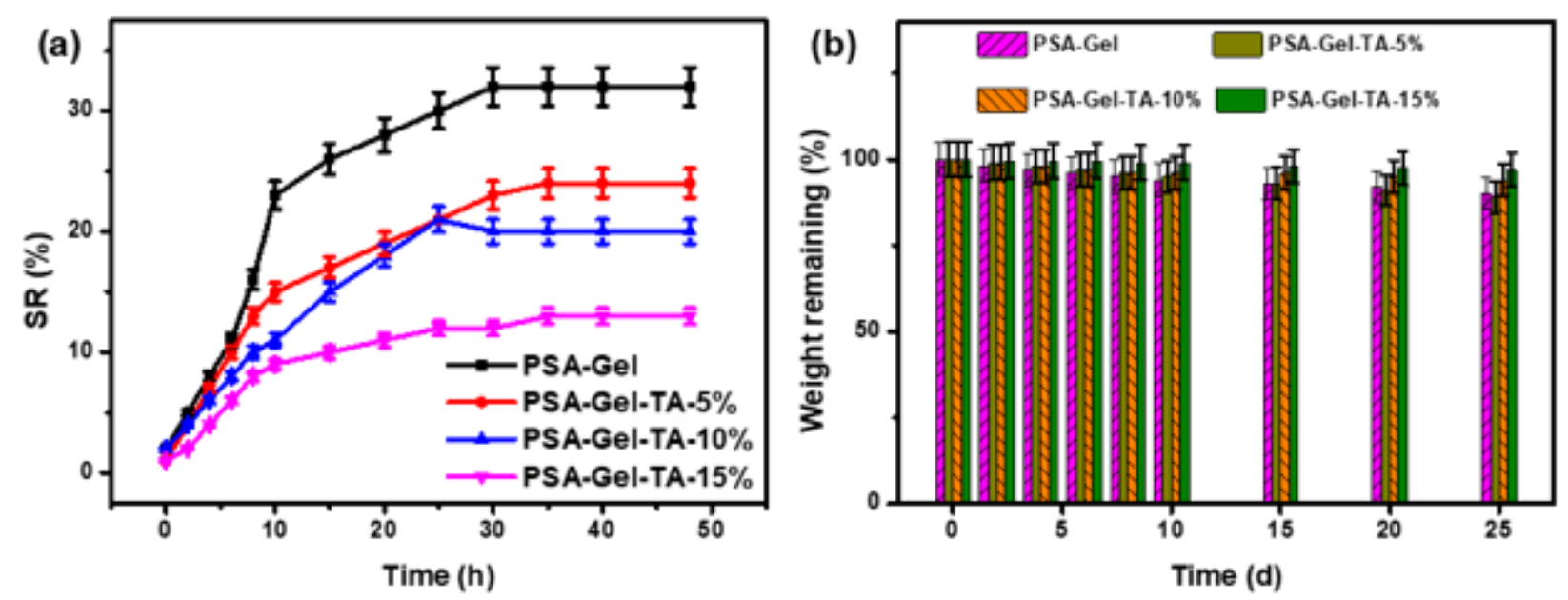

Figure 3

(A) Swelling kinetics of PSA-Gel and PSA-Gel-TA hydrogels in the PBS $(\mathrm{pH}=7.4)$ within $50 \mathrm{~h}$. (B) Degradation curves of PSA-Gel and PSA-Gel-TA hydrogels in the PBS $(\mathrm{pH}=7.4)$ within 21 days.
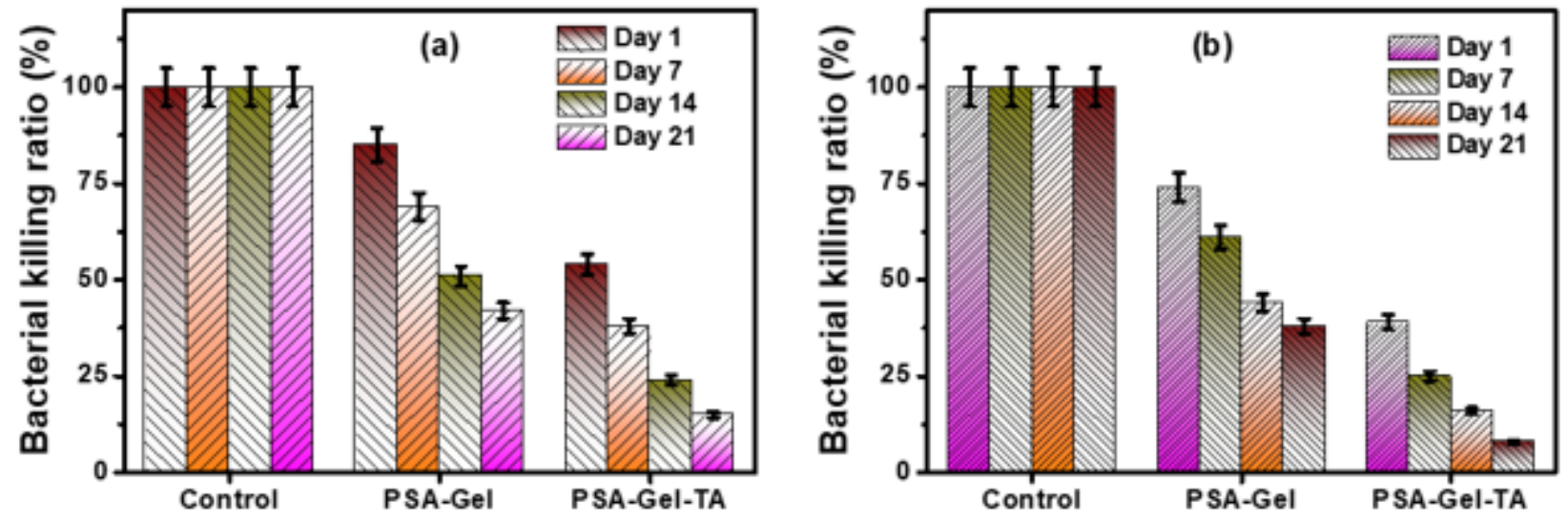

Figure 4

Bacterial survival ratios of $\mathrm{S}$. aureus (a) and E. coli (b) as a function of day 1 to days 21 for the control, PSA-Gel and PSA-Gel-TA hydrogels. 

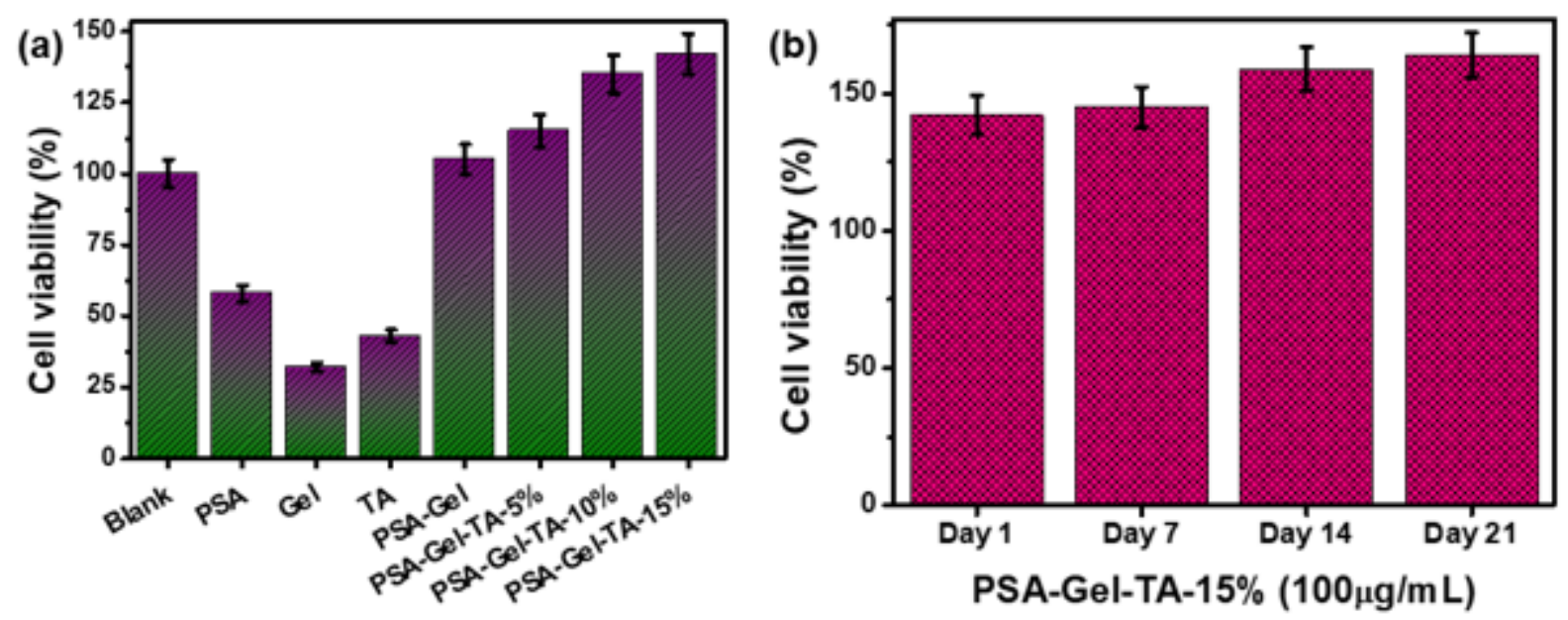

Figure 5

The L929 cell viability of the (a) blank, PSA, Gel, TA, PSA-Gel hydrogel, PSA-Gel-TA-5\%, PSA-Gel-TA-10\%, and PSA-Gel-TA-15\% hydrogels $(100 \mu \mathrm{g} / \mathrm{mL})$ and (b) Cell viability of PSA-Gel-TA- $15 \%$ hydrogels (100 $\mu \mathrm{g} / \mathrm{mL}$ ) as a function of day 1 to days 21 measured using MTT assay.

(a) Day 7

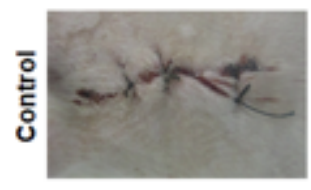

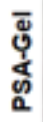

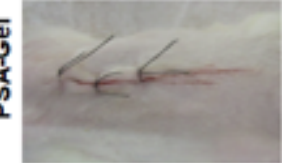

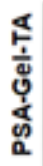

Day 14
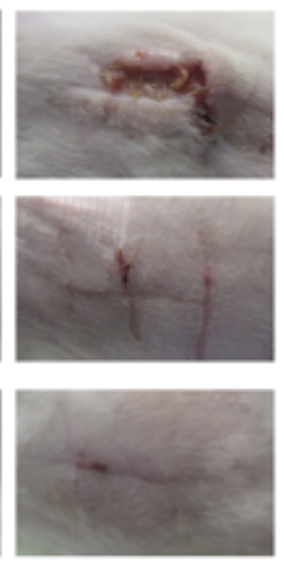

Day 21

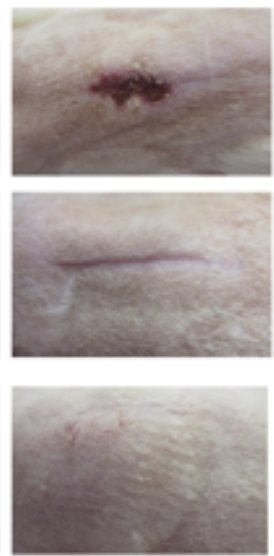

(b)

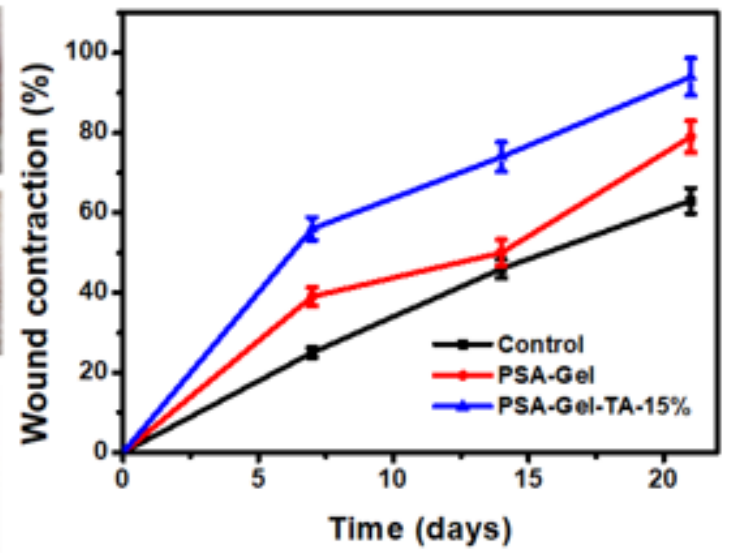

Figure 6

(a) Representative photographs of the wound 7, 14, and 21 days after full-thickness skin excision and (b) The percentage of wound contraction of control, PSA-Gel hydrogel and PSA-Gel-TA hydrogels wound dressings. 

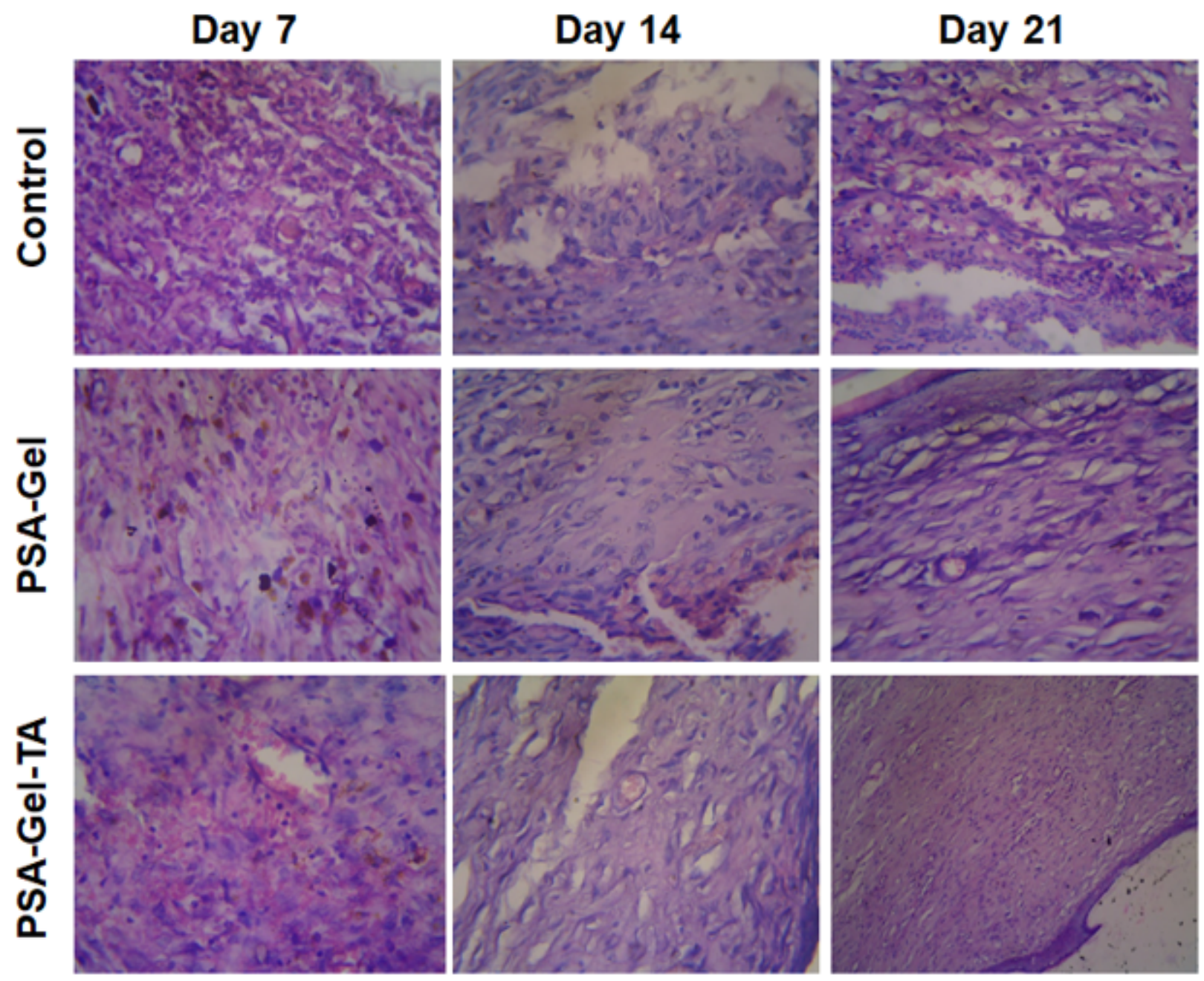

Figure 7

Representative histological images (100x) of skin wounds untreated, Na PGO 10\% and Ca PGO 10\% wound dressings. GT: granulation tissue; IC: inflammatory cells; EP: epidermis: DE: dermis.

\section{Supplementary Files}

This is a list of supplementary files associated with this preprint. Click to download.

- GraphicalAbstract.docx 